\title{
DEVELOPMENT OF THE SCRF POWER COUPLER FOR THE APT ACCELERATOR
}

\author{
Eric N. Schmierer, Richard E. Lujan, Brian Rusnak, Brian Smith, W. B. Haynes, Cort Gautier, \\ J. A. Waynert, Frank Krawczyk, Los Alamos National Laboratory, Los Alamos, NM, \\ Jack Gioia, General Atomics, Los Alamos, NM
}

\begin{abstract}
The team responsible for the design of the Accelerator Production of Tritium (APT) superconducting (SC) radio frequency (RF) power coupler has developed two 700$\mathrm{MHz}$, helium gas-cooled power couplers. One has a fixed inner conductor and the other has an adjustable inner conductor (gamma prototype and alpha prototype). The power couplers will be performance tested in the near future. This paper discusses the mechanical design and fabrication techniques employed in the development of each power coupler. This includes material selection, copper coating, assembly sequences, and metal joining procedures, as well as the engineering analyses performed to determine the dynamic response of the inner conductors due to environmental excitations. A bellows is used in both prototype inner conductors in the area near the ceramic RF window, to compensate for thermal expansion and mechanical tolerance build-up. In addition, a bellows is used near the tip of the inner conductor of the alpha prototype for tuning the power coupler after it is installed on the accelerator. Extensive analytical work has been performed to determine the static loads transmitted by the bellows due to thermally induced expansion on the inner conductor and on the RF window. This paper also discusses this analysis, as well as the mechanical analysis performed to determine the final geometric shape of the bellows. Finally, a discussion of the electromagnetic analysis used to optimize the performance of the power couplers is included.
\end{abstract}

\section{INTRODUCTION}

The superconducting portion of the $1100 \mathrm{MeV}$ APT accelerator will have numerous five-cell cavities. Each cavity will require two power couplers, which will transmit up to $420 \mathrm{~kW}$ of continuous RF power at a 700 Mhz frequency. RF power is transmitted to the accelerator from a klystron via WR 1500 waveguide at atmospheric pressure. A waveguide-to-coaxial conductor transition will be located near the RF cavity. Accelerator vacuum will be maintained through the coaxial conductor by a planar coaxial ceramic window located in the coaxial conductor portion of the window assembly. Figure 1 shows a cutaway view of a power coupler and a vacuum window assembly on a cryomodule.

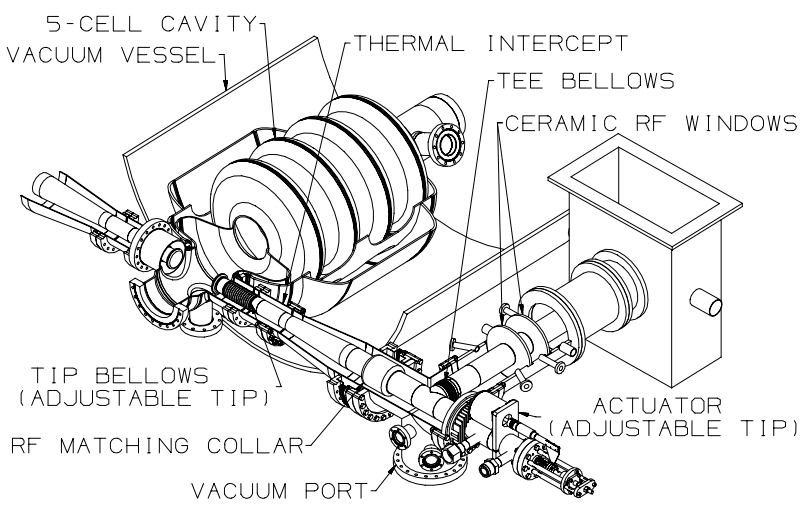

Figure 1: Power Coupler, Window, Cryomodule Assembly

\section{RF ANALYSIS}

The $420 \mathrm{~kW}$ RF power is delivered to each five-cell cavity by two power couplers. To ensure a reliable longterm operation for this plant-type facility, an attempt was made to create a power coupler design using features and components suggested by recent experience gained at other accelerator laboratories. These features include planar coaxial windows (a standard in high-power klystrons), coaxial couplers (successfully operated at highpower, e.g., at DESY [1]), positioning of the RF windows outside the line-of-sight of the proton beam, an additional vacuum-pump port in the quarter wave stub region for a good vacuum at the windows (recent experience at KEK [2]), and provisions for a simple cooling scheme of the center conductor. These were integrated into a new power coupler design. To achieve this design, extensive threedimensional electromagnetic modeling has been done with the MAFIA electromagnetic simulator [3]. The modeling procedure started with the design of separate components and benchmarking the simulations by building and measuring some of these components [4]. Then a fully integrated study was performed with interaction with RF window fabricators.

The simulations investigated several variations of subcomponents (various quarter-wave stubs and matching devices), and multiple iterations with mechanical and thermal evaluations. The thermal evaluation of the RF power deposited onto the inner and outer conductor was derived from the three-dimensional RF simulations. Figure 2 shows the MAFIA analytical model of the quarter wave stub. 


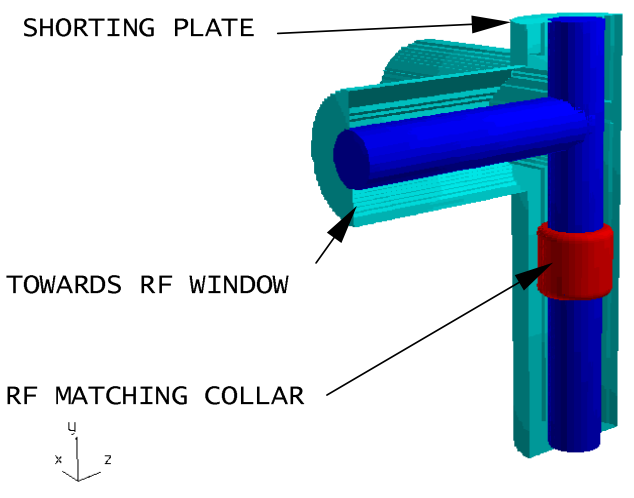

Figure 2: MAFIA Analytical Model

\section{THERMAL ANALYSIS}

A 200-node, axisymmetric model using the finite difference approximation was made to describe the inner and outer conductors. The model includes grey body; diffuse; infra-red radiation exchange; conduction through stainless steel, copper, and niobium; RF heating (travelling or standing wave power distributions); and convection cooling of the inner and outer conductors.

Detailed results for the inner and outer conductors have been published [5]. The inner conductor is found to be adequately cooled for both travelling and standing waves by using $300 \mathrm{~K}$ helium at 1.2 atmospheres pressure, flowing at $3 \mathrm{~g} / \mathrm{s}$ through a 3-mm annular space formed by a stainless-steel sleeve within the copper inner conductor.

The outer conductor cooling approaches considered distributed counter-flow and localized thermal intercepts. The selected cooling scheme was the result of an extensive trade-study which considered: integration of the power coupler cooling into the cryomodule; impact on the cryoplant and distribution system; room temperature refrigeration input power; and manufacturability, maintainability, and reliability issues. The selected configuration is a double point thermal intercept where the low temperature thermal intercept inlet fluid is $4.6 \mathrm{~K}, 12$ bar helium. After exiting the low temperature intercept, the supercritical helium is used to intercept heat loads from the beam tube, thermal shield, and structural supports. It is then circulated through the power-coupler high-temperature intercept. The fluid is returned to the cryoplant at about $30 \mathrm{~K}$.

\section{MECHANICAL DESIGN}

The WR 1500 waveguide-to-coaxial conductor transition is of the tee-bar type and it is water or air cooled. The transition is integrated into the RF window assembly. The coolant(s) is also circulated inside the inner conductor section of the RF window assembly to the interface with the power coupler. Cooling air is also circulated between the ceramic windows. The coaxial conductor is nominally 6.125 in. diameter from the tee-bar location through the ceramic window and the quarter-wave stub, to an area that is approximately $17.25 \mathrm{in}$. from the shorting plate. At this location, the coaxial conductor tapers down to nominally 4.06-in. diameter through a distance of 8.44 in. The coaxial conductor remains this size through the interface of the power coupler with the five-cell cavity. The inner conductor is supported at the shorting plate and is cantilevered through a distance of approximately $36.43 \mathrm{in}$. The center of the RF matching collar is located $11.87 \mathrm{in}$. away from the shorting plate. A nominal 2.60-in. diameter bellows is used at the intersection of the two coaxial conductors, which comprise the quarter-wave stub geometry. The adjustable inner conductor has a nominal 1.71-in. diameter bellows located near its tip. The RF window assembly connects to the inner conductor at a reentrant receiver located at the interface. The conductive material for the inner conductor is oxygen-free electronic grade copper, ASTM F68-93 class 2 or better. The material for the receiver and both bellows is beryllium copper, B194 alloy 25 . The $300-\mathrm{K}$ helium is delivered to the intake plenum located at the rear of the inner conductor. Inside the plenum, the flow is orificed and split so that some of the coolant is circulated through a stainless-steel liner towards the tip. The coolant is returned through the annular space formed by the outside of the stainless-steel liner and the inside of the copper tube. The beryllium copper receiver and bellows in the quarter-wave stub region are cooled with the coolant flowing through a small supply tube that provides helium directly onto the bottom of the receiver and flows down the inside of the bellows. Figure 3 shows the inner conductor coolant flow path.

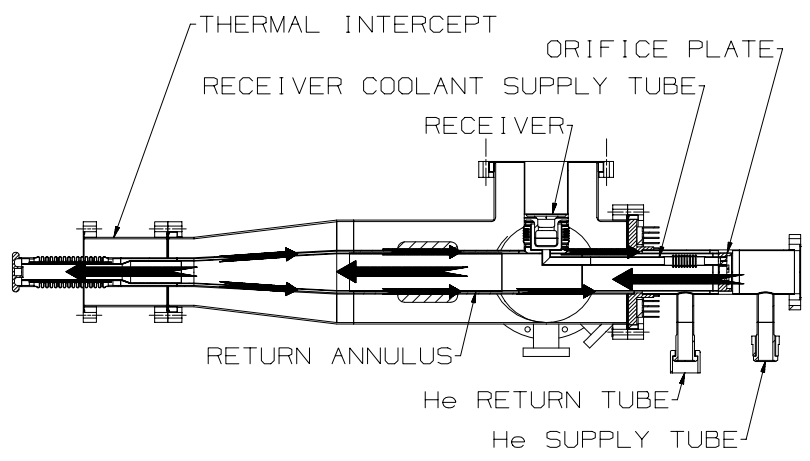

Figure 3: He Coolant Flow Path

All copper-to-copper joining for the inner conductor is specified as e-beam welding. To expedite fabrication, however, tungsten-inert gas (TIG) welding with appropriate final machining was approved. All copper-tostainless steel joints are furnace brazed. The outer conductor material is stainless steel type 304, whose conductive surfaces are electroplated with copper per specification APT-CG-RFPC-001. The double point thermal intercept is integrated with the outer conductor as a flanged spool piece. Metal seal flanges are used at all 
$\mathrm{RF} / \mathrm{vacuum}$ connections with modified copper gaskets to enhance RF integrity. A vacuum pumping port is located in the vicinity of the quarter wave stub. The RF analysis indicates that the geometry of the port is such that an RF grill is not needed at this location.

Using COSMOS/M Ver. 2.0 linear dynamic module, a finite-element model was developed and fundamental modes of vibration calculated for the inner conductor. The first four were $39.7 \mathrm{~Hz}, 55.9 \mathrm{~Hz}, 180.5 \mathrm{~Hz}$, and $187.4 \mathrm{~Hz}$ for the tip of the gamma power coupler. For the tip of the alpha power coupler, they were $29.96 \mathrm{~Hz}, 35.71 \mathrm{~Hz}, 101.6$ $\mathrm{Hz}$, and $206.4 \mathrm{~Hz}$, with an assumed damping coefficient of 0.01 . The harmonic analysis of the tip resulted in a $Q$ of 40. The first source of excitation directly attached to the alpha power coupler is the $250 \mathrm{l} / \mathrm{s}$ vacuum pump. The specified displacement of the vacuum pump applied to the shorting plate resulted in a RMS displacement of the tip of $1.6 \times 10^{-5} \mathrm{~m}$. Using a random vibration excitation previously obtained from another accelerator facility, the RMS displacement of the tip was $3 \times 10^{-6} \mathrm{~m}$.

The force required to make the RF joint at the interface with the RF window assembly was large enough to warrant mechanical testing. Two receivers were tested to 4200 lbs., resulting in no detectable yielding of the receiver material.

\section{BELLOWS DEVELOPMENT}

The inner conductor is constrained by the ceramic vacuum windows and the shorting plate located on the quarterwave stub. The tee-bellows must accommodate thermally induced expansion and any misalignments of the assembly when docking the vacuum window assembly to the power coupler, so the ceramic windows must react any forces transmitted by the bellows. Maximum allowable forces on the ceramic windows of 3-lb. axial force and 10-lb. lateral force were set as design goals. Based on anticipated thermally induced expansion and installation compression, spring rates of $150 \mathrm{lb}$./in. maximum axial and $1000 \mathrm{lb}$./in. maximum lateral are required of the bellows. This assumes no mechanical assembly preloads. The tip bellows allows adjustment of the inner conductor tip. The tip-bellows spring rate was specified at $150 \mathrm{lbs}$./in. maximum axial, with a compression of 0 in. to 0.6 in.

Bellows convolution shape was determined from RF analysis and was optimized for fabrication and spring rate requirements. Three materials were pursued. They are Be$\mathrm{Cu}$, electro-formed $\mathrm{Cu}$, and $\mathrm{Cu}$-plated stainless steel. Be$\mathrm{Cu}$ was the primary choice because of its superior mechanical properties. An electro-formed copper bellows was the second choice. Type II electro-formed copper has a better yield strength than OFE $\mathrm{Cu}$ but is still susceptible to work hardening, and there are issues with outgassing, weldability, and consistency in the bellows convolution thickness. Other electro-form alloys and configurations were considered but were not pursued. $\mathrm{Cu}$ plated stainless steel would add the process of brazing $\mathrm{Cu}$ end-rings for welding to the assembly and would add the issues associated with the plating process. It is unknown whether thermal and mechanical cycling, high power RF, or electron-beam welding would degrade the plating, create Ni flashing interference, or affect the plating adhesion.

\section{CONCLUSION}

The engineering and experimental effort has yielded a power coupler design that analysis indicates has a power transmission of better than $-40 \mathrm{~dB}$ at or around the 700$\mathrm{MHz}$ operation frequency. Figure 4 shows the broad band transmission/reflection curve of the power coupler taken from electromagnetic simulations. Fabrication of three gamma prototype power couplers has been completed, and they are scheduled to be tested on the Room Temperature Test Bed [6] in the near future.

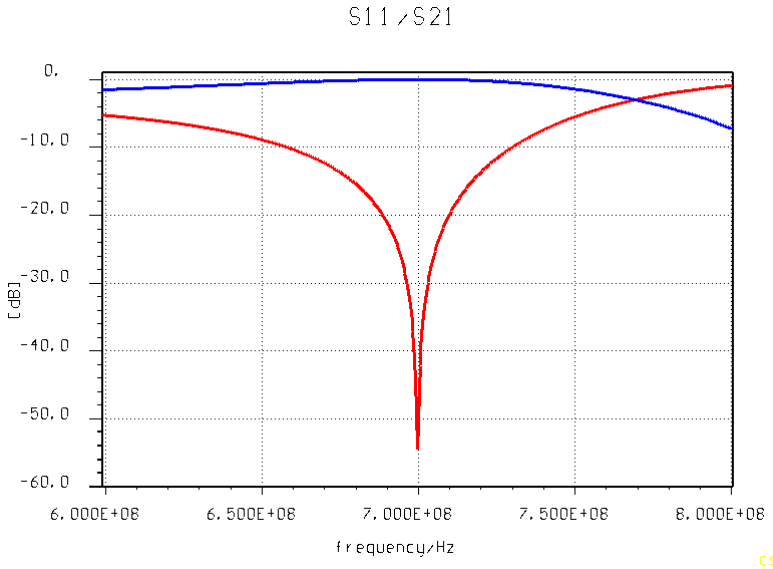

Figure 4: Transmission/Reflection Curve

\section{REFERENCES}

[1] B. Dwersteg, DESY, "Progress of TTF High Power Input Coupler Design at DESY,", Proceedings of the 8th RF Superconductivity workshop, Abano Terme, Italy, (1997).

[2] S. Mitsunobu, KEK, "High Power Input Coupler and Windows,', Proceedings of the 8th RF Superconductivity workshop, Abano Terme, Italy, (1997).

[3] The MAFIA Collaboration, "User Guide,", CST Gmbh, Darmstadt, Germany.

[4] Frank Krawczyk, LANL, "Electromagnetic Modeling of Cavities and Power Couplers for SC High-Current Proton Accelerators," Invited Talk at the APS Spring Meeting, Columbus, Ohio, (1998).

[5] J.A. Waynert, F.C. Prenger, "A Thermal Analysis and Optimization of the APT $210 \mathrm{~kW}$ Power Coupler," Proceedings of LINAC98, Aug. 1998, Chicago, IL.

[6] J. Gioia, General Atomics et.al, "A Room Temperature Test Bed for Evaluating 700-MHz RF Windows and Power Couplers for the Superconducting Portion of the APT Accelerator," Proceedings of PAC' 99, New York, NY, (1999). 